\title{
Excellent Schottky Characteristics of Indium-tin-oxide Contact to n-type GaN
}

\author{
Kyurin Kim, Youngun Gil, and Hyunsoo Kim* \\ School of Semiconductor and Chemical Engineering, Semiconductor Physics Research Center, \\ Chonbuk National University, \\ Jeonju 561-756, Republic of Korea
}

\begin{abstract}
Excellent Schottky characteristics of indium-tinoxide (ITO) contact formed on n-type GaN (n-GaN) were demonstrated. The post-thermal annealing of ITO contact sputtered on n-GaN led to a significant improvement in the Schottky characteristics, particularly pronounced in the air ambient than nitrogen ambient, e.g., the rectification ratio (measured at $\pm 1.0 \mathrm{~V}$ ) was increased from 4.9 to 4240 after an optimized post-thermal annealing. Thermionic field emission model applied to the forward current-voltage curves of the ITO/n-GaN Schottky diodes also exhibited that the Schottky barrier height could be as high as $0.93 \mathrm{eV}$. The observed excellent Schottky characteristics with postthermal annealing are attributed to the improved ITO crystallinity as verified by glancing $\mathrm{X}$-ray diffraction method
\end{abstract}

Keywords-indium tin oxide; Schottky contact; thermal annealing; thermionic field emission.

\section{INTRODUCTION}

Indium tin oxide (ITO) has been very widely used as the transparent conductive electrodes in organic and inorganic optoelectronic devices, particularly, in the light emitting diodes (LEDs) and solar cells because of its high optical transmittance in the visible wavelength and good electrical conductivity.[1-3] In GaN-based LEDs, ITO deposited on $\mathrm{p}-\mathrm{GaN}$ is commercially used as the transparent conductive electrodes to spread the current more uniformly.[4-8] Due to this reason, a number of studies with respect to ITO contacts to $\mathrm{p}-\mathrm{GaN}$ have been presented.[4-8] For example, Margalith et al.[4] showed that the ITO Ohmic contact to p-GaN could not be easily obtained due to the large work-function mismatch between ITO $(4.6 \mathrm{eV})$ and $\mathrm{p}-\mathrm{GaN}(7.5 \mathrm{eV})$, and more importantly, due to the sputtering ion damage produced at the $\mathrm{p}-\mathrm{GaN}$ surface such as nitrogen vacancies and oxygen impurities acting as donors.[9] Therefore, ITO materials are usually deposited on p-GaN using an e-beam evaporator to avoid sputtering ion damage[10] and/or the modified p-GaN structures[11] such as strained layer or superlattices are used instead.

Despite such depth insight into ITO/p-GaN contact, the studies on ITO/n-GaN contact are very lacking.[12,13] This might be due to the lack of possible application of ITO/n-GaN contact system. However, it is worth noting that, if the ITO produces Ohmic contact to n-GaN, ITO can be used as the n-type transparent conductive electrodes of GaN-based LEDs, in which may lead to a enhancement of light extraction efficiency. Furthermore, ITO Ohmic contact can be also applicable in the photoswitching devices. Meanwhile, if the ITO yields non-
Ohmic contact to n-GaN, namely, Schottky characteristics, ITO can be practically applicable in the photodiodes. These feasibilities indicate that the studies on the behavior of ITO contact to n-GaN are of particular interest. In this regard, we have investigated the electrical properties of ITO contact formed on n-type GaN as a function of postthermal annealing conditions. It is shown that the ITO contact produces excellent Schottky characteristics after post-thermal annealing in air ambient.

\section{EXPERIMENTAL PROCEDURE}

A $2.8 \mu \mathrm{m}$-thick $n$-GaN (Si-doped)/1.95 $\mu$ m-thick undoped GaN wafers grown on c-plane sapphire substrates by metal-organic chemical vapor deposition (MOCVD) were used for the contact study. Hall-effect measurements revealed that the carrier concentration $N=1.6 \times 10^{18} \mathrm{~cm}^{-3}$, the Hall mobility $\mu=221 \mathrm{~cm}^{2} \mathrm{~V}^{-1} \mathrm{~s}^{-1}$, and the sheet resistance $R_{\mathrm{sh}}=55.3 \Omega / \mathrm{sq}$. For the fabrication of Schottky diodes, first, the Ohmic metal pad was formed on the wafer using a Ti/Al $(30 \mathrm{~nm} / 70 \mathrm{~nm})$ layers, followed by rapid thermal annealing performed at $550{ }^{\circ} \mathrm{C}$ for $1 \mathrm{~min}$ in $\mathrm{N}_{2}$ ambient. Note that, prior to the metal deposition, the sample surface was cleaned with the buffered oxide etchant for $5 \mathrm{~min}$ to remove native oxide and rinsed with deionized water. The conventional photolithography and e-beam evaporation techniques were used for the Ohmicmetal formation. Then, the $100 \mathrm{~nm}$-thick ITO layer was deposited on the entire wafer using a radio frequency magnetron sputtering system, followed by a selective wetchemical etching of ITO layer to form Schottky electrodes. The ITO sputtering was performed using the following condition, i.e., the composition of ITO target was 90 weight $\% \mathrm{In}_{2} \mathrm{O}_{3}$ and 10 weight $\% \mathrm{SnO}_{2}$, the working pressure was $10 \mathrm{mTorr}, \mathrm{Ar} / \mathrm{O}_{2}$ gas ratio was 10:0, the $\mathrm{rf}$ power was $100 \mathrm{~W}$, and the growth temperature was 500 ${ }^{\circ} \mathrm{C}$. Hall-effect measurement of as-deposited ITO layer showed that $N=9.5 \times 10^{20} \mathrm{~cm}^{-3}, \mu=7.4 \mathrm{~cm}^{2} \mathrm{~V}^{-1} \mathrm{~s}^{-1}$, and the electrical resistivity is $8.6 \times 10^{-4} \Omega \mathrm{cm}$. The completed device structure with a circular Schottky contact (diameter $=50 \mu \mathrm{m}$ ) is shown in the inset of Fig. 1. To investigate the effect of post-thermal annealing, the devices were annealed at the temperatures of $400-800{ }^{\circ} \mathrm{C}$ for $1 \mathrm{~min}$ in nitrogen and air ambient. The electrical characteristics of ITO contact were evaluated using a parameter analyzer (HP4156A). The structural changes with post-thermal annealing were investigated using glancing X-ray diffraction (XRD) measurements. 

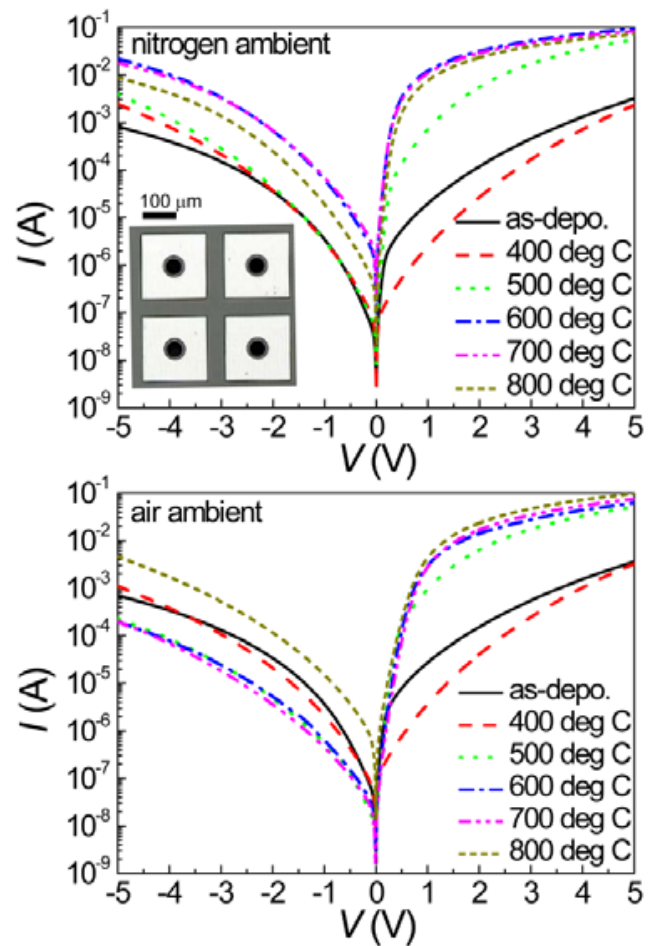

Fig.1. Typical semi-logarithmic $I-V$ characteristics of the ITO contact to $\mathrm{n}-\mathrm{GaN}$ plotted as a function of annealing temperature and ambient. The inset shows the optical microscopic top-view of the fabricated diodes.

\section{RESULTS AND DISCUSSION}

Figure 1 shows the typical semi-logarithmic $I-V$ characteristics of the ITO contact to $\mathrm{n}-\mathrm{GaN}$ plotted as a function of annealing temperature and ambient. Note that the as-deposited ITO contact produces slightly rectifying behavior, indicating that the ITO Ohmic contact to n-GaN cannot be easily obtained in spite of high $N$ values of nlayer. Indeed, this result is somewhat different as compared with that of Sheu et al.,[12] reported that the asdeposited ITO produced Ohmic contact to n-GaN. The observed different behavior might be attributed to the different ITO deposition condition, i.e., the growth temperature of ITO was as high as $500{ }^{\circ} \mathrm{C}$, in which high growth temperature is expected to influence the ITO crystallinity, as will be discussed later in details.

Interestingly, the post-thermal annealing led to a significant increase in the forward currents without regard to annealing ambient. This can be attributed to the improved electrical conductivity of ITO layer, e.g., the ITO layer annealed at $700{ }^{\circ} \mathrm{C}$ for $1 \mathrm{~min}$ in air ambient showed the reduced electrical resistivity of $3.5 \times 10^{-4} \Omega \mathrm{cm}$. Furthermore, for the sample annealed in $\mathrm{N}_{2}$ ambient, the reverse currents also increased after thermal annealing, which seems to be negative effect in terms of rectifying behavior. In contrast, for the sample annealed in air ambient, the reverse current decreased with thermal annealing up to $700{ }^{\circ} \mathrm{C}$, while that increased significantly above $800{ }^{\circ} \mathrm{C}$. This indicates that the rectifying behavior of ITO contact to n-GaN can be significantly improved by the post-thermal annealing performed in air ambient.

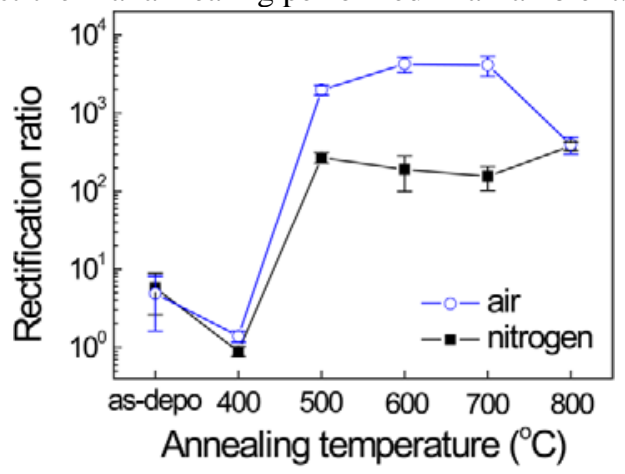

Fig.2. The rectification ratio of ITO contact to $n-G a N$ as measured at \pm 1 V.

Figure 2 shows the rectification ratio of ITO contact to $\mathrm{n}$-GaN, as measured at $\pm 1 \mathrm{~V}$. As expected, the rectification ratio increased significantly with increasing annealing temperature, particularly pronounced at 600$700{ }^{\circ} \mathrm{C}$. Notably, the samples annealed in air ambient always yielded higher rectification ratios than those annealed in nitrogen ambient. Consequently, the very high rectification ratio of $\sim 4200$ could be obtained for the samples annealed at $600-700{ }^{\circ} \mathrm{C}$ in air ambient.

To analyze the transport mechanism of ITO contact/nGaN system, the forward $I-V$ curves were analyzed using a thermionic field emission (TFE) theory[14,15] because the calculated characteristic tunneling parameter $\left(E_{00}\right)$ of 1.46 lies in the TFE regime, i.e., $k T / q E_{00} \sim 1$ for TFE, where, $k$ is the Boltzmann constant, $T$ is the temperature, $q$ is the electronic charge. Here, the $E_{00}$ was estimated according to

$$
E_{00}=\frac{q h}{4 \pi}\left(\frac{N}{\varepsilon_{s} m^{*}}\right)^{1 / 2}
$$

where $h$ is the Planck constant, $\varepsilon_{\mathrm{s}}$ is the dielectric constant of the semiconductor $\left(\varepsilon_{\mathrm{s}}=8.9 \varepsilon_{0}\right)$, and $m^{*}$ is the electron effective mass $\left(m^{*}=0.2 m_{\mathrm{e}}\right)$. Thereby, the forward $I-V$ curves can be fitted with TFE model, i.e., $[14,15]$

$$
I=A \frac{A^{\prime \prime} T \sqrt{\pi E_{0} e\left(\Phi_{B}-V-\xi\right)}}{k \cosh \left(E_{\infty} / k T\right)} \exp \left[-\frac{e \xi}{k T}-\frac{q}{E_{00} \operatorname{coth}\left(E_{00} / k T\right)}\left(\Phi_{B}-\xi\right)\right] \exp \left[\frac{q V}{E_{\infty 0} \operatorname{coth}\left(E_{00} / k T\right)}\right]
$$

where $\mathrm{A}$ is the contact area, $\mathrm{A}^{* *}$ is the Richardson constant (26.4 A/cm2K2), ФB is the effective Schottky barrier height, and $\xi$ is the energy difference between the conduction band edge and Fermi level. 
TABLE 1. SCHOTTKY PARAMETERS OF ITO CONTACT TO N-GAN ANNEALED IN AIR AMBIENT.

\begin{tabular}{cccc}
\hline$T\left({ }^{\circ} \mathrm{C}\right)$ & $E_{00}(\mathrm{eV})$ & $n$ & $\Phi_{\mathrm{B}}(\mathrm{eV})$ \\
\hline 0 & 0.217 & 8.12 & 1.08 \\
400 & 0.206 & 7.71 & 1.13 \\
500 & 0.047 & 1.86 & 0.78 \\
600 & 0.208 & 2.49 & 0.93 \\
700 & 0.201 & 2.41 & 0.91 \\
800 & 0.244 & 2.90 & 0.92 \\
\hline
\end{tabular}

Table I summarizes the Schottky parameters including the E00, ideality factor (n), and $\Phi B$. Here, note that the $n$ value, which was calculated using the relation of $\mathrm{n}=(\mathrm{E} 00 / \mathrm{kT}) \operatorname{coth}(\mathrm{E} 00 / \mathrm{kT})$, was much larger than 1.0 for all cases, indicating that non-ideal carrier transport occurred at the ITO contact interface. According to previous studies,[15, 16] carrier transport through surface states associated with point defects such as nitrogen vacancies or oxygen interstitials (generated during ITO sputtering) or threading dislocations might be responsible for the nonideal transport at the contact interface. Notably, however, the $n$ value decreased significantly with post-thermal annealing, indicating that the density of surface states were reduced. It is also worth noting that the $\Phi B$ of optimized ITO contact (annealed at $600{ }^{\circ} \mathrm{C}$ ) is as high as $0.93 \mathrm{eV}$, which is much larger than the theoretically predicted value of $0.5 \mathrm{eV}$. This suggests that the thermodynamic reactions of ITO/n-GaN with thermal annealing led to an increase in the barrier height.

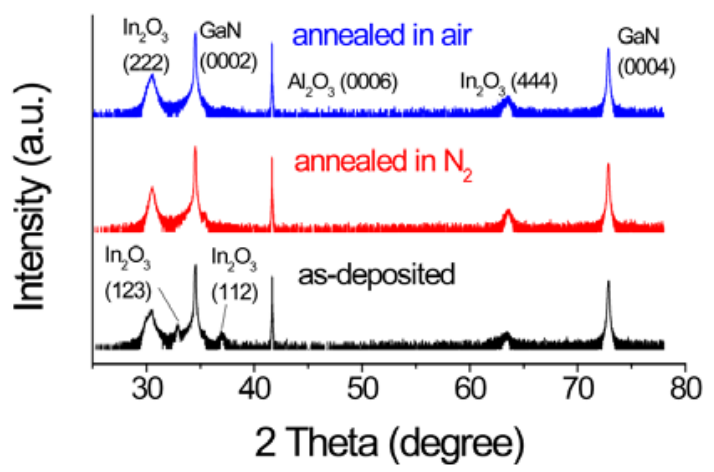

Fig.3. XRD $(\theta-2 \theta)$ peaks of the as-deposited and $700{ }^{\circ} \mathrm{C}$ annealed samples.

To support this hypothesis, XRD $(\theta-2 \theta)$ measurements were performed for the as-deposited sample and $700{ }^{\circ} \mathrm{C}$ annealed sample in nitrogen and air ambient, as shown in Fig. 3. Notably, the XRD peaks of In2O3 (222) and (444) planes increased significantly after thermal annealing, while those of In2O3 (123) and (112) planes disappeared. These results indicate that the post thermal annealing resulted in a preferential regrowth of In2O3 (222) and (444) planes associated with improved crystallinity of ITO films. The degree of improved ITO crystallinity could be estimated by comparing the peak intensity of In2O3 (222) relative to the GaN (0002), namely, the normalized peak intensity ratio of In2O3 (222)/GaN (0002) was $0.74,1.37$, and 1.92 for the as-deposited ITO,
ITO annealed in nitrogen, and air ambient, respectively, which is consistent with the Schottky characteristics. Therefore, the improved Schottky behavior with thermal annealing, particularly in air ambient, is due to the formation of ideal ITO-GaN interfaces accompanied with the regrowth of In2O3 (222) and (444) planes or improved crystallinity of ITO films.

\section{CONCLUSION}

To summarize, excellent ITO Schottky contact to nGaN could be demonstrated by post thermal annealing in air ambient. The rectification ratio could be as high as 4200 after optimized post-thermal annealing condition, which was associated with the increased forward currents and the suppressed reverse currents. TFE model showed that the ideality factor was significantly decreased with post-thermal annealing, indicating a formation of ideal ITO/n-GaN interfaces. The Schottky barrier height could be alos obtained to be as high as $0.93 \mathrm{eV}$. XRD analysis clearly showed that the improved Schottky characteristics would be correlated to the formation of ideal ITO-GaN interfaces accompanied with the regrowth of In2O3 (222) planes or improved crystallinity of ITO films. These results suggest that the ITO would be used as the transparent Schottky electrodes for photodiodes or optical sensors.

\section{ACKNOWLEDGEMENTS}

This research was financially supported in part by the Ministry of Education and National Research Foundation of Korea (NRF) through the Human Resource Training Project for Regional Innovation (2013H1B8A2032197), and in part by Basic Science Research Program through the National Research Foundation of Korea(NRF) funded by the Ministry of Education, Science and Technology (2014R1A1A1A05007455).

\section{REFERENCES}

[1] Ishibashi, S., Higuchi, Y. \& Nakamura, K., Low resistivity indiumtin oxide transparent conductive films. II. Effect of sputtering voltage on electrical property of films. J. Vac. Sci. Technol. A, 8(3), pp.1403-1406, 1990.

[2] Ishida, T., Kobayashi, H. \& Nakato, Y., Structures and properties of electron-beam-evaporated indium tin oxide films as studied by $\mathrm{x}$-ray photoelectron spectroscopy and work-function measurements. Journal of Applied Physics, 73(9), pp.4344-4350, 1993.

[3] Kim, H., Gilmore, C. M., Horwitz, J. S., Mattoussi, H., Murate, H., Kafafi, S. H. \& Chrisey, D. B., Electrical, optical, and structural properties of indium-tin-oxide thin films for organic light-emitting devices. Journal of Applied Physics, 86(11), pp. 6451-6461, 1999.

[4] Margalith, T., Buchinsky, O., Cohen, D. A., Abare, A. C., Hansen, M., DenBarrs, S. P. \& Coldren, L. A., Indium tin oxide contacts to gallium nitride optoelectronic devices. Applied Physics Letters, 74(26), pp.3930-3932, 1999.

[5] Saitoh, M., Saito, T., Inukai, T. \& Hiramoto, T., Transport spectroscopy of the ultrasmall silicon quantum dot in a singleelectron transistor. Applied Physics Letters, 79(18), pp. 2025-2927, 2001. 
[6] Kim, D.W, Sung, Y. J., Park, J. W. \& Yeom, G. Y., A study of transparent indium tin oxide (ITO) contact to $\mathrm{p}-\mathrm{GaN}$. Thin solid Films, 398-399, pp.87-92, 2001.

[7] Kim, J. K., Chhajed, S., Schubert, M. F., Schubert, E. F. Fischer, A. J., Crawford, M. H., Cho, J., Kim, H. \& Sone, C., Light-Extraction Enhancement of GaInN Light-Emitting Diodes by GradedRefractive-Index Indium Tin Oxide Anti-Reflection Contact. Advanced Materials, 20(4), pp.801-804, 2008.

[8] Choi, Y. \& Kim, H., Surface Fermi level pinning and carrier transport of indium-tin-oxide Ohmic contact to p-type GaN. Journal of Alloys and Compounds, 533, pp.15-18, 2012.

[9] Cao, X. A., Pearton, S. J., Zhang, A. P., Dang, G. T., Ren, F., Shul, R. J., Zhang, L., Hickman, R. \& Van Hove, J. M., Electrical effects of plasma damage in p-GaN. Applied Physics Letters, 75(17), pp.2569-2571, 1999.

[10] Kim, K. K., Kim, H., Lee, S. N. \& Cho, S., Structural, Optical, and Electrical Properties of E-Beam and Sputter-Deposited ITO Film for LED Applications. Electronic Materials Letters, 7(2), pp.145149, 2011

[11] Song, J. O., Ha. J. S. \& Seong, T. Y., Ohmic-Contact Technology for GaN-Based Light-Emitting Diodes: Role of P-Type Contact. Electron Devices, IEEE Transactions on, 57(1), pp.42-59, 2010

[12] Sheu, J. K., Su, Y. K., Chi, G.C., Jou, M. J. Chang, \& C. M., Effects of thermal annealing on the indium tin oxide Schottky contacts of n-GaN. Appl. Phys. Lett. 72(25), pp. 3317-3319, 1998 ; Sheu, J. K., Su, Y. K., Chi, G. C., Jou, M. J., Liu, C. C. \& Chang, C. M., Indium tin oxide ohmic contact to highly doped n-GaN. SolidState Electron. 43(11), pp. 2081-2084, 1999

[13] Shim, K. H., Paek, M. C., Lee, B. T., Kim, C. \& Kang, J. Y., Preferential regrowth of indium-tin oxide(ITO) films deposited on GaN(0001) by rf-magnetron sputter. Applied Physics A, 72(4), pp.471-474, 2001

[14] Padovani, F. A. \& Stratton, R. Field and thermionic-field emission in Schottky barriers. Solid-state Electronics, 9(7), pp.695-707, 1966

[15] Jang, J. S., Kim, D. \& Seong, T. Y., Schottky barrier characteristics of Pt contacts to n-type InGaN, Journal of Applied Physics, 99(7), pp.073704(1-4), 1999.

[16] Kim, H., Ryou, J. H., Dupuis, R. D., Lee, S. N., Park, Y. Jeon, J. W. \& Seong, T. Y., Electrical characteristics of contacts to thin film Npolar n-type GaN, Applied Physics Letters, 93(19), pp.192106(1-3), 2008 